\title{
Condensate fraction of a Fermi gas in the BCS-BEC crossover
}

\author{
Luca Salasnich and Nicola Manini \\ Dipartimento di Fisica and INFM, Università di Milano, Via Celoria 16, 20133 Milano, Italy
}

Alberto Parola

Dipartimento di Fisica e Matematica and INFM, Università dell'Insubria, Via Valleggio 11, 22100 Como, Italy

(Received 31 May 2005; published 30 August 2005)

\begin{abstract}
We investigate the Bose-Einstein condensation of fermionic pairs in a uniform two-component Fermi gas, obtaining an explicit formula for the condensate density as a function of the chemical potential and the energy gap. We analyze the condensate fraction in the crossover from the Bardeen-Cooper-Schrieffer (BCS) state of weakly interacting Cooper pairs to the Bose-Einstein condensate of molecular dimers. By using the localdensity approximation we study confined Fermi vapors of alkali-metal atoms for which there is experimental evidence of condensation also on the BCS side of the Feshbach resonance. Our theoretical results are in agreement with these experimental data and give the behavior of the condensate on both sides of the Feshbach resonance at zero temperature.
\end{abstract}

DOI: 10.1103/PhysRevA.72.023621

Several experimental groups have reported the observation of the crossover from the Bardeen-Cooper-Schrieffer (BCS) state of weakly bound Fermi pairs to the BoseEinstein condensate (BEC) of molecular dimers with ultracold two-hyperfine-component Fermi vapors of ${ }^{40} \mathrm{~K}$ atoms [1-3] and ${ }^{6} \mathrm{Li}$ atoms $[4,5]$. The detection of a finite condensed fraction also in the BCS side of the crossover $[2,4]$ stimulated a debate over its interpretation [6-10]. Extended BCS (EBCS) equations [11-13] have been used to reproduce in a satisfactory way density profiles [14] and collective oscillations [15] of these Fermi gases. As this EBCS mean-field theory is defined for any value of the coupling, it provides an interpolation between the BCS weak-coupling regime and the BEC strong-coupling limit [16,17]. Despite well-known limitations [16] the EBCS theory is considered a reliable approximation for studying the whole BCS-BEC crossover at zero temperature, giving a simple and coherent description of the crossover in terms of fermionic variables.

Within the EBCS scheme, we derive an explicit formula for the number of condensed fermionic pairs in the uniform BCS ground state. We use the EBCS equations to study the behavior of this condensate fraction as a function of the interatomic scattering length $a_{F}$ in the BCS-BEC crossover: from the BCS regime (small negative $a_{F}$ ) crossing the unitarity limit (infinitely large $a_{F}$ ) to the BEC regime (small positive $a_{F}$ ). In addition, by using the local-density approximation, we calculate the condensate fraction and density profiles of the Fermi gas in anisotropic harmonic confinement. With no fitted parameters, we find a remarkable agreement with recent experimental results [4] indicating a relevant fraction of condensed pairs of ${ }^{6} \mathrm{Li}$ atoms also on the BCS side of the Feshbach resonance.

The Hamiltonian density of a dilute interacting two-spincomponent Fermi gas in a box of volume $V$ is given by

$$
\hat{\mathcal{H}}=-\frac{\hbar^{2}}{2 m} \sum_{\sigma=\uparrow, \downarrow} \hat{\psi}_{\sigma}^{\dagger} \nabla^{2} \hat{\psi}_{\sigma}+g \hat{\psi}_{\uparrow}^{\dagger} \hat{\psi}_{\downarrow}^{\dagger} \hat{\psi}_{\downarrow} \hat{\psi}_{\uparrow}
$$

where $\hat{\psi}_{\sigma}(\mathbf{r})$ is the field operator that destroys a fermion of spin $\sigma$ in the position $\mathbf{r}$, while $\hat{\psi}_{\sigma}^{\dagger}(\mathbf{r})$ creates a fermion of spin
PACS number(s): 03.75.Hh, 03.75.Ss

$\sigma$ in $\mathbf{r}$. The attractive interatomic interaction is modeled by a contact pseudopotential of strength $g(g<0)$. The field operators satisfy the usual anticommutation rules and can be expanded in Fourier series, $\hat{\psi}_{\sigma}(\mathbf{r})=V^{-1 / 2} \sum_{\mathbf{k}} e^{i \mathbf{k} \cdot \mathbf{r}} \hat{a}_{\mathbf{k} \sigma}$, in terms of operators $\hat{a}_{\mathbf{k} \sigma}$ destroying a fermion of spin $\sigma$ with linear momentum $\hbar \mathbf{k}$. At zero temperature the BCS variational state [18] is given by $|\varphi\rangle=\Pi_{\mathbf{k}}\left(u_{k}+v_{k} \hat{a}_{\mathbf{k} \uparrow}^{\dagger} \hat{a}_{-\mathbf{k} \downarrow}^{\dagger}\right)|0\rangle$, where $|0\rangle$ is the vacuum state, $u_{k}$ and $v_{k}$ are variational amplitudes, and the state $|\varphi\rangle$ is normalized to unity if $u_{k}^{2}+v_{k}^{2}=1$. The amplitudes $u_{k}$ and $v_{k}$ are obtained by imposing the minimization of the thermodynamic potential

$$
\Omega=\int d^{3} \mathbf{r}\langle\hat{\mathcal{H}}(\mathbf{r})-\mu \hat{n}(\mathbf{r})\rangle,
$$

where $\hat{n}(\mathbf{r})=\Sigma_{\sigma=\uparrow, \downarrow} \hat{\psi}_{\sigma}^{\dagger}(\mathbf{r}) \hat{\psi}_{\sigma}(\mathbf{r})$ is the number density operator and $\mu$ is the chemical potential, determined by the condition $N=\int d^{3} \mathbf{r}\langle\hat{n}(\mathbf{r})\rangle$ that fixes the average number $N$ of fermions. All averages are done over the BCS state $|\varphi\rangle$. Accordingly, the standard BCS equation for the number of particles is

$$
N=2 \sum_{\mathbf{k}} v_{k}^{2},
$$

and for the BCS gap is

$$
-1 / g=(1 / V) \sum_{\mathbf{k}}\left(1 / 2 E_{k}\right)
$$

$[18,19]$. Here

$$
E_{k}=\left[\left(\epsilon_{k}-\mu\right)^{2}+\Delta^{2}\right]^{1 / 2}
$$

and

$$
v_{k}^{2}=\frac{1}{2}\left[1-\left(\epsilon_{k}-\mu\right) / E_{k}\right]
$$

with the noninteracting fermion kinetic energy $\epsilon_{k}$ $=\hbar^{2} k^{2} /(2 m)$. The chemical potential $\mu$ and the gap energy $\Delta$ are obtained by solving Eqs. (3) and (4). Unfortunately, in the continuum limit, due to the choice of a contact potential, the gap equation diverges in the ultraviolet. A suitable regularization is obtained by introducing the scattering length $a_{F}$ via the equation 


$$
m / 4 \pi \hbar^{2} a_{F}=(1 / g)+(1 / V) \sum_{\mathbf{k}}\left(1 / 2 \epsilon_{k}\right),
$$

and then subtracting this equation from the gap equation [11-13]. In this way one obtains the regularized gap equation

$$
-m / 4 \pi \hbar^{2} a_{F}=(1 / V) \sum_{\mathbf{k}}\left(1 / 2 E_{k}-1 / 2 \epsilon_{k}\right) .
$$

The EBCS equations (3) and (8) can be used to study the evolution from BCS superfluidity of Cooper pairs $\left(a_{F}<0\right)$ to the Bose-Einstein condensation of molecular dimers $\left(a_{F}>0\right)[11-13,16]$. This transition is conveniently studied as a function of the dimensionless inverse interaction parameter $y=\left(k_{F} a_{F}\right)^{-1}$, where $k_{F}=\left(3 \pi^{2} n\right)^{1 / 3}$ is the Fermi wave vector of noninteracting fermions of the same density [20-22].

As previously stressed, several properties of ultracold Fermi gases have been investigated in the last few years by using the EBCS equations $[14,15]$. Here we analyze the condensate fraction of fermionic pairs that is strictly related to the off-diagonal long-range order (ODLRO) [23] of the system. As shown by Yang [24], the BCS state $|\varphi\rangle$ guarantees the ODLRO of the Fermi gas, namely, that, in the limit wherein both unprimed coordinates approach an infinite distance from the primed coordinates, the two-body density matrix factorizes as follows:

$$
\left\langle\hat{\psi}_{\uparrow}^{\dagger}\left(\mathbf{r}_{1}^{\prime}\right) \hat{\psi}_{\downarrow}^{\dagger}\left(\mathbf{r}_{2}^{\prime}\right) \hat{\psi}_{\downarrow}\left(\mathbf{r}_{1}\right) \hat{\psi}_{\uparrow}\left(\mathbf{r}_{2}\right)\right\rangle=\left\langle\hat{\psi}_{\uparrow}^{\dagger}\left(\mathbf{r}_{1}^{\prime}\right) \hat{\psi}_{\downarrow}^{\dagger}\left(\mathbf{r}_{2}^{\prime}\right)\right\rangle\left\langle\hat{\psi}_{\downarrow}\left(\mathbf{r}_{1}\right) \hat{\psi}_{\uparrow}\left(\mathbf{r}_{2}\right)\right\rangle .
$$

The largest eigenvalue $N_{0}$ of the two-body density matrix (9) gives the number of Fermi pairs in the lowest state, i.e., the condensate number of Fermi pairs [24,25]. This number is given by

$$
N_{0}=\int d^{3} \mathbf{r}_{1} d^{3} \mathbf{r}_{2}\left|\left\langle\hat{\psi}_{\downarrow}\left(\mathbf{r}_{1}\right) \hat{\psi}_{\uparrow}\left(\mathbf{r}_{2}\right)\right\rangle\right|^{2},
$$

and it is straightforward to show [25] that

$$
N_{0}=\sum_{\mathbf{k}} u_{k}^{2} v_{k}^{2}
$$

It is quite remarkable that such a formula can be expressed in a simple form as a function of the chemical potential $\mu$ and the energy gap $\Delta$. In fact, in the continuum limit $\Sigma_{\mathbf{k}} \rightarrow V /(2 \pi)^{3} \int d^{3} \mathbf{k} \rightarrow V /\left(2 \pi^{2}\right) \int k^{2} d k$, taking into account the functional dependence (6) of the amplitudes $u_{k}$ and $v_{k}$ on $\mu$ and $\Delta$, we find

$$
n_{0}=N_{0} / V=\left(m^{3 / 2} / 8 \pi \hbar^{3}\right) \Delta^{3 / 2} \sqrt{\mu / \Delta+\sqrt{1+\mu^{2} / \Delta^{2}}} .
$$

By the same techniques, also the two EBCS equations can be written in a more compact form as

$$
\begin{aligned}
-1 / a_{F} & =\left[2(2 m)^{1 / 2} / \pi \hbar^{3}\right] \Delta^{1 / 2} I_{1}(\mu / \Delta), \\
n=N / V & =\left[(2 m)^{3 / 2} / 2 \pi^{2} \hbar^{3}\right] \Delta^{3 / 2} I_{2}(\mu / \Delta),
\end{aligned}
$$

where $I_{1}(x)$ and $I_{2}(x)$ are two monotonic functions which can be expressed in terms of elliptic integrals, as shown by Marini, Pistolesi, and Strinati [26].

Simple analytical results from Eqs. (12)-(14) can be obtained in three limiting cases: (i) the BCS regime, where

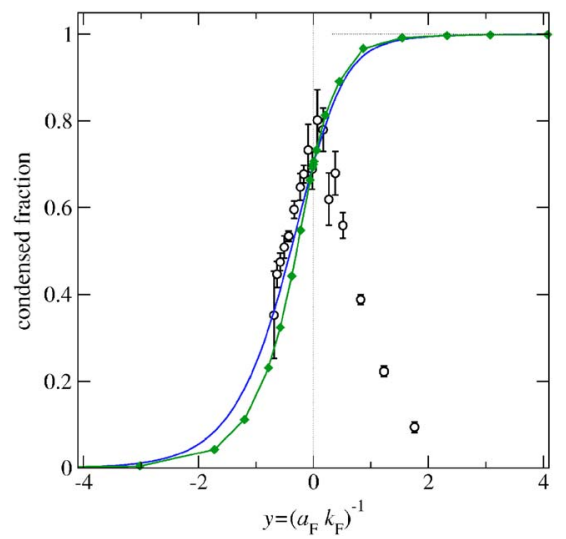

FIG. 1. (Color online) Condensate fraction $N_{0} /(N / 2)$ of Fermi pairs in the uniform two-component dilute Fermi gas as a function of $y=\left(k_{F} a_{F}\right)^{-1}$ (solid line). The same quantity computed in the local-density approximation for a droplet of $N=6 \times 10^{6}$ fermions in an elongated harmonic trap with $\omega_{\perp} / \omega_{z}=47$, as in the experiment of Ref. [4], plotted against the value of $y$ at the center of the trap (joined diamonds). Open circles with error bars: experimentally determined condensed fraction [4].

$y \ll-1, \mu / \Delta \gg 1$, and the size of weakly bound Cooper pairs exceeds the typical interparticle spacing $k_{F}^{-1}$; (ii) the unitarity limit, with $y=0$ (thus $a_{F}= \pm \infty$ ); (iii) the BEC regime, where $y \gg 1, \mu / \Delta \ll-1$ and the fermions condense as a gas of tight dimers.

(i) BCS regime. $\mu$ approaches the noninteracting Fermi energy $\epsilon_{F}=\hbar^{2} k_{F}^{2} /(2 m)$. One finds that $I_{1}(x) \simeq \sqrt{x}[\ln (8 x)-2]$ and $I_{2}(x) \simeq 2 x^{3 / 2} / 3$. It follows that the condensate density is given by

$$
n_{0} \simeq\left(m k_{F} / 8 \pi \hbar^{2}\right) \Delta=\left(3 \pi / 2 e^{2}\right) n \exp [(\pi / 2) y]
$$

with an exponentially small energy gap $\Delta$ $=8 e^{-2} \epsilon_{F} \exp (y \pi / 2)$.

(ii) Unitarity limit. $I_{1}(x)=0$ and $I_{2}(x) \simeq 1.16$ with $x$ $=\mu / \Delta \simeq 0.85$. In addition one finds $\mu \simeq 0.59 \epsilon_{F}$ and $\Delta$ $\simeq 0.69 \epsilon_{F}$, and the condensate fraction is

$$
2 N_{0} / N \simeq 0.6994 \text {. }
$$

(iii) $B E C$ regime. The condensate fraction approaches the ideal Bose gas value $2 N_{0} / N \simeq 1$, with all pairs (molecular dimers) moving into the condensate. In addition one finds that $I_{1}(x) \simeq-\pi \sqrt{|x|} / 2$ and $I_{2}(x) \simeq \pi /(8 \sqrt{|x|})$. From these expressions we obtain

$$
n_{0} \simeq n / 2 \simeq\left(m^{2} a_{F} / 8 \pi \hbar^{4}\right) \Delta^{2},
$$

which is precisely the condensate density deduced by Pieri and Strinati in this BEC regime using a Green-function formalism [27]. In this limit the chemical potential approaches half the dimer binding energy $\mu \simeq-\hbar^{2} /\left(2 m a_{F}^{2}\right)=-\epsilon_{F} y^{2}$ and the gap $\Delta \simeq 4(3 \pi)^{-1 / 2} \epsilon_{F} y^{1 / 2}$.

The behavior of the condensate fraction $2 N_{0} / N$ through the BCS-BEC crossover is shown in Fig. 1 (solid line) as a function of the Fermi-gas parameter $y$. The figure shows that a large condensate fraction builds up in the BCS side already before the unitarity limit, and that on the BEC side it rapidly converges to the ideal boson gas value. The finding of a finite 
nontrivial $2 N_{0} / N \neq 1$ around the unitarity limit contrasts with the suggestion [6] that the condensate fraction could reach unity already at $y=0$.

The local-density approximation (LDA) can be implemented to calculate the properties of a nonuniform Fermi gas of sufficiently slowly varying density, as in the case of a large number $N$ of particles in a smooth trapping potential $U(\mathbf{r})$. In the spirit of the Thomas-Fermi method, we replace the chemical potential $\mu$ with the quantity $\mu-U(\mathbf{r})$ into Eqs. (12)-(14). In this way the energy gap $\Delta(\mathbf{r})$, the total density $n(\mathbf{r})$, and the condensate density $n_{0}(\mathbf{r})$ become local scalar fields. In order to compare with the experiment of Ref. [4] done with ${ }^{6} \mathrm{Li}$ atoms, we solve the LDA equations for $N=6 \times 10^{6}$ fermions trapped in an external anisotropic harmonic potential well defined by a transverse frequency $\omega_{\perp}$ and an axial frequency $\omega_{z}$ :

$$
U(\mathbf{r})=(m / 2)\left[\omega_{\perp}^{2}\left(x^{2}+y^{2}\right)+\omega_{z}^{2} z^{2}\right] .
$$

We take an anisotropy ratio $\omega_{\perp} / \omega_{z}=47$. For given $a_{F}$, and for a given initial guess of $\mu$, the local chemical potential $\mu-U(\mathbf{r})$ is computed and Eq. (13) for $\Delta$ is solved numerically, and this calculation is repeated for each $\mathbf{r}$ on a spatial grid. The total number $N$ of fermions in the trap is then computed by integrating Eq. (14) over the position $\mathbf{r}$. The $\mu$ parameter is then adjusted iteratively in order to obtain the required total number $N$ of fermions within the droplet. The final density, gap, and $n_{0}$ functions are determined according to Eqs. (12)-(14).

We find that at the border of the droplet, all three of these functions vanish together, for both positive and negative $a_{F}$. Figure 2 reports the computed axial density

$$
n_{z}(z)=\int d x d y n(x, y, z)
$$

and condensed density $2 n_{0 z}(z)$ (defined analogously) for three values of the scattering length $a_{F}$. The fermionic cloud is rather diffuse in the BCS region, and gets more and more compact as the BEC regime is approached. The smoothly vanishing behavior of $n_{z}(z)$ near the cloud border is due to the integration over transverse coordinates: $n(\mathbf{r})$ instead vanishes with a finite jump in its gradient component across the surface. The same applies for the condensed quantities. Of course, this sharp vanishing behavior is a consequence of the LDA, while quantum delocalization effects (here neglected) should smooth the density profile at the surface of the actual droplet.

As shown in Fig. 2, the condensed fraction (dotted curve) vanishes as one moves from center to border of the BCS cloud, as pairing acts less efficiently as the density is low. On the contrary, on the BEC side, the condensed fraction increases to unity toward the cloud border, as the bosonic gas suffers less from quantum depletion wherever its density is smaller. In the unitarity limit, the condensed fraction is in fact constant, and equals the uniform value of Eq. (16): this reflects the scale invariance of this special limit of infinitely large scattering length.
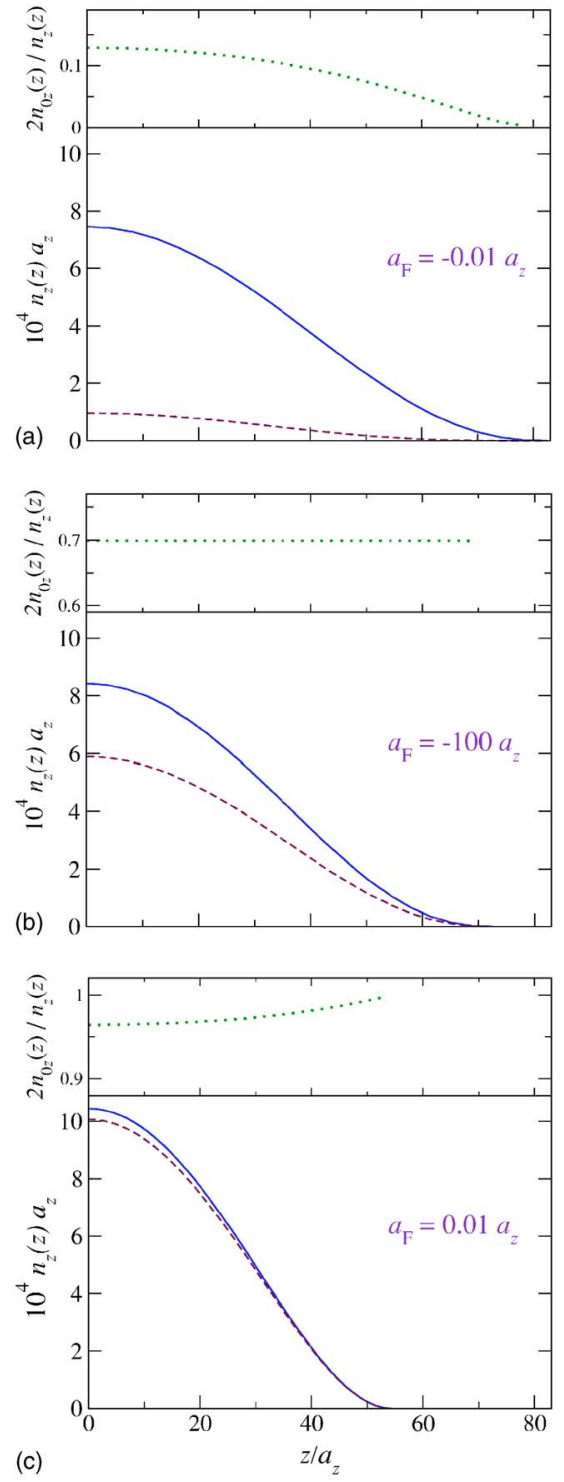

FIG. 2. (Color online) Axial total density $\left[n_{z}(z)\right.$, solid $]$ and condensed density $\left[2 n_{0 z}(z)\right.$, dashed] of a droplet composed of $N=6$ $\times 10^{6}$ fermions in an elongated harmonic trap with $\omega_{\perp} / \omega_{z}=47$, as in the experiment of Ref. [4]. With ${ }^{6} \mathrm{Li}$ atoms, the harmonic oscillator length scale $a_{z}=\hbar^{1 / 2}\left(m \omega_{z}\right)^{-1 / 2}=12 \mu \mathrm{m}$ in the setup of Ref. [4]. Dotted curves: the axial local condensate fraction $2 n_{0 z}(z) / n_{z}(z)$. The values of $y$ at the center of the droplet are $-1.2\left(a_{F}=-0.01 a_{z}\right)$, $-10^{-4}\left(a_{F}=-100 a_{z}\right)$, and $0.87\left(a_{F}=0.01 a_{z}\right)$, representative of the $\mathrm{BCS}$, unitarity, and BEC regimes, respectively.

The experiment of Ref. [4] addresses the problem of measuring the total condensate fraction of trapped interacting fermions near the unitarity limit. Even though it would be hard to demonstrate that the measured "condensed fraction" coincides with the ODLRO $2 N_{0} / N$ defined in Eq. (10), we attempt a comparison of the computed total condensed fraction, reported in Fig. 1, with the experimental data at the lowest temperature $k_{B} T=0.05 \epsilon_{F}$. In the experiment the scattering length is tuned by means of an external magnetic tuned across a Feshbach resonance. Following Ref. [28], the scattering length $a_{F}$ as a function of the magnetic field $B$ near the Feshbach resonance is given by 


$$
a_{F}=a_{b}\left[1+\alpha\left(B-B_{0}\right)\right]\left[1+B_{r} /\left(B-B_{0}\right)\right],
$$

where $B_{0}=83.4149 \mathrm{mT}, a_{b}=-1405 a_{0}, B_{r}=30.0 \mathrm{mT}$, and $\alpha=0.0040(\mathrm{mT})^{-1}$. The measured condensed fraction at the lowest temperature is reported in Fig. 1 as open circles with error bars. The general increasing trend and the substantially large value of the condensed fraction on the BCS side and close to the unitarity limit agree quite well with the EBCS LDA calculation (joined diamonds). On the BEC side the rapid drop of experimental data is due to inelastic losses and thus these data are not reliable [4]. Note also that the condensate fraction measured near a Feshbach resonance of ${ }^{40} \mathrm{~K}$ atoms [2] is much smaller than the values reported in Ref. [4]. The origin of this discrepancy has not been clarified yet $[4,6-9]$.

As suggested in the Introduction, the zero-temperature EBCS theory has some limitations. In particular, it overestimates the bosonic scattering length $a_{B}$ between molecular dimers in the BEC regime: the theory predicts $a_{B}=2 a_{F}$, where $a_{F}$ is the two-fermion scattering length, but Monte Carlo results [20] confirm the four-body scattering analysis [29] which gives $a_{B}=0.6 a_{F}$. To overcome such difficulties, the EBCS theory can be further extended by including beyond mean-field corrections which improve the determination of the chemical potential, and reduce the bosonic scattering length to $a_{B}=0.75 a_{F}$ [30].

A finite-temperature formulation of the EBCS mean-field theory is not difficult $[19,31]$ : for the condensed number of Fermi pairs we find the formula $N_{0}=\Sigma_{\mathbf{k}} u_{k}^{2} v_{k}^{2} \tanh ^{2}\left(\beta E_{k} / 2\right)$, where $\beta=1 /\left(k_{B} T\right)$. It is then straightforward to express this relation as an explicit function of $\mu, \Delta$, and $\beta$, pretty much like Eqs. (12) at $T=0$. On the other hand, it is well known $[13,14,16,17]$ that in the BEC regime the finitetemperature mean-field theory overestimates the critical $T_{c}$.
This is related to the dimer breaking energy rather than the loss of coherence of the bosonic gas as it should. As a consequence, the condensed fraction is overestimated near the unitarity limit and in the BEC region: to get reasonable results beyond mean-field corrections are needed $[13,14,16,17,32]$.

In this paper we have shown that the zero-temperature EBCS mean-field theory gives a simple and nice formula for the condensed fraction of Fermi pairs in the full BCS-BEC crossover. On the BCS side of the Feshbach resonance our theoretical results are in agreement with the low-temperature measurements of Zwierlein et al. [4]. In fact, the quantitative agreement of the measured and computed condensate fraction (based on the off-diagonal long-range-order parameter) is no demonstration that these two quantities can be identified. A quantitative microscopic simulation of the experimental procedure could in fact rigorously prove or disprove the identification we are putting forward in the present work. Such a simulation should be based on a microscopic theory which contains an essentially correct internal dynamics of the pairs across the crossover: this goes likely beyond the capability of a mean-field method such as EBCS. Also alternative experimental techniques could be developed to provide an independent measurement of the condensed fraction, possibly in closer contact with the off-diagonal long-range order. Further work, both experimental and theoretical, could help to elucidate this interesting issue.

On the BEC side of the Feshbach resonance the experimental data of Ref. [4] are not reliable due to inelastic losses. In this region our predictions may be a guide for further experimental investigations.

The authors acknowledge D. Pini and L. Reatto for enlightening discussions, and C. E. Campbell for useful email suggestions.
[1] M. Greiner et al., Nature (London) 426, 537 (2003).

[2] C. A. Regal et al., Phys. Rev. Lett. 92, 040403 (2004).

[3] J. Kinast et al., Phys. Rev. Lett. 92, 150402 (2004).

[4] M. W. Zwierlein et al., Phys. Rev. Lett. 92, 120403 (2004); M. W. Zwierlein et al., ibid. 94, 180401 (2005).

[5] C. Chin et al., Science 305, 1128 (2004); M. Bartenstein et al., Phys. Rev. Lett. 92, 203201 (2004).

[6] G. M. Falco and H. T. C. Stoof, Phys. Rev. Lett. 92, 130401 (2004).

[7] A. V. Avdeenkov and J. L. Bohn, Phys. Rev. A 71, 023609 (2005).

[8] R. B. Diener and T. L. Ho, e-print cond-mat/0404517.

[9] A. Perali et al., e-print cond-mat/0501631.

[10] G. Ortiz and J. Dukelsky, e-print cond-mat/0503664.

[11] D. M. Eagles, Phys. Rev. 186, 456 (1969).

[12] A. J. Leggett, in Modern Trends in the Theory of Condensed Matter, edited by A. Pekalski and J. Przystawa (Springer, Berlin, 1980), p. 13.

[13] P. Noziéres and S. Schmitt-Rink, J. Low Temp. Phys. 59, 195 (1985).

[14] A. Perali et al., Phys. Rev. A 68, 031601(R) (2003); 93, 100404 (2004).

[15] H. Hu et al., Phys. Rev. Lett. 93, 190403 (2004).
[16] C. A. R. Sa de Melo et al., Phys. Rev. Lett. 71, 3202 (1993).

[17] J. R. Engelbrecht et al., Phys. Rev. B 55, 15153 (1997).

[18] J. Bardeen et al., Phys. Rev. 108, 1175 (1957).

[19] A. L. Fetter and J. D. Walecka, Quantum Theory of ManyParticle Systems (McGraw-Hill, New York, 1971).

[20] G. E. Astrakharchik et al., Phys. Rev. Lett. 93, 200404 (2004).

[21] H. Heiselberg, Phys. Rev. Lett. 93, 040402 (2004).

[22] N. Manini and L. Salasnich, Phys. Rev. A 71, 033625 (2005).

[23] O. Penrose, Philos. Mag. 42, 1373 (1951); O. Penrose and L. Onsager, Phys. Rev. 104, 576 (1956).

[24] C. N. Yang, Rev. Mod. Phys. 34, 694 (1962).

[25] C. E. Campbell, in Condensed Matter Theories Vol. 12, edited by J. W. Clark and P. V. Panat (Nova Science, New York, 1997), p. 131.

[26] M. Marini et al., Eur. Phys. J. B 1, 151 (1998).

[27] P. Pieri and G. C. Strinati, Phys. Rev. Lett. 91, 030401 (2003).

[28] M. Bartenstein et al., Phys. Rev. Lett. 94, 103201 (2005).

[29] D. S. Petrov et al., Phys. Rev. Lett. 93, 090404 (2004).

[30] P. Pieri and G. C. Strinati, Phys. Rev. B 61, 15370 (2000).

[31] L. D. Landau et al., Statistical Physics, Part 2, Course of Theoretical Physics (Butterworth-Heinemann, New York, 1980), Vol. 9.

[32] H. Hu et al., e-print cond-mat/0506046. 